\title{
PATHOLOGY
}

\section{Gleason score 7 prostate cancer on needle biopsy: relation of primary pattern 3 or 4 to pathological stage and progression after radical prostatectomy}

Amin A, Partin A, Epstein JI

Department of Pathology, The Johns Hopkins Medical Institutions, Baltimore, Maryland, USA

J Urol. 2011; 186: 1286-90

Purpose: There have been only a few contradictory publications assessing whether Gleason score $4+3=7$ has a worse prognosis than $3+4=7$ on biopsy material in predicting pathological stage and biochemical recurrence. Older studies predated the use of the modified Gleason grading system established in 2005.

Materials and Methods: We retrospectively studied 1,791 cases of Gleason score 7 on prostatic biopsy to determine whether the breakdown of Gleason score 7 into $3+4$ vs $4+3$ has prognostic significance in the modern era.

Results: There was no difference in patient age, preoperative serum prostate specific antigen, maximum tumor percent per core or the number of positive cores between Gleason score $3+4=7$ and Gleason score $4+$ $3=7$. Gleason score $4+3=7$ showed an overall correlation with pathological stage (organ confined, focal extraprostatic extension, nonfocal extraprostatic extension, seminal vesicle invasion/lymph node metastases, $\mathrm{p}=0.005)$. On multivariate analysis Gleason score $4+3=7(\mathrm{p}=0.03)$, number of positive cores $(\mathrm{p}=0.002)$, maximum percent of cancer per core $(\mathrm{p}=0.006)$ and preoperative serum prostate specific antigen $(\mathrm{p}=0.03)$ all correlated with pathological stage. Gleason score $4+3=7$ on biopsy was also associated with an increased risk of biochemical progression after radical prostatectomy $(\mathrm{p}=0.0001)$. On multivariate analysis Gleason score $4+3=7(p=0.001)$, maximum percent of cancer per core $(p<0.0001)$ and preoperative serum prostate specific antigen $(p<0.0001)$ but not number of positive cores correlated with the risk of biochemical progression after radical prostatectomy.

Conclusions: Our study further demonstrates that Gleason score 7 should not be considered a homogenous group for the purposes of disease management and prognosis.

\section{Editorial Comment}

The importance of grading prostate carcinoma is evidenced by the fact that it is included in all nomograms used to predict pathologic stage and biochemical progression following radical prostatectomy. The Gleason score may be used to define prognostic groups.

The most frequent combination of Gleason scores defining prognostic groups is 2-4, 5-6, 7, and 8-10. Gleason score 7 may result from $3+4$ or $4+3$ patterns. There is conflicting data as to the prognostic difference of Gleason score 7 on a biopsy depending on whether the primary pattern is 3 or $4(1,2)$.

The study from the Johns Hopkins based on a very large number of patients showed that Gleason score 7 should not be considered a homogeneous group for the purpose of disease management and prognosis. 
There was no difference in patient age, preoperative serum prostate specific antigen, maximum tumor percent per core or the number of positive cores between Gleason score $3+4=7$ and Gleason score $4+3=7$. However, Gleason score $4+3=7$ showed a statistically significant correlation with pathological stage, and on multivariate analysis an increased risk for biochemical progression following radical prostatectomy.

\section{References}

1. Makarov DV, Sanderson H, Partin AW, Epstein JI: Gleason score 7 prostate cancer on needle biopsy: is the prognostic difference in Gleason scores $4+3$ and $3+4$ independent of the number of involved cores? J Urol. 2002; 167: 2440-2.

2. Grober ED, Tsihlias J, Jewett MA, Sweet JM, Evans AJ, Trachtenberg J, et al.: Correlation of the primary Gleason pattern on prostate needle biopsy with clinico-pathological factors in Gleason 7 tumors. Can J Urol. 2004; 11: 2157-62.

Dr. Athanase Billis

Full-Professor of Pathology

State University of Campinas, Unicamp

Campinas, São Paulo, Brazil

E-mail:athanase@fcm.unicamp.br 